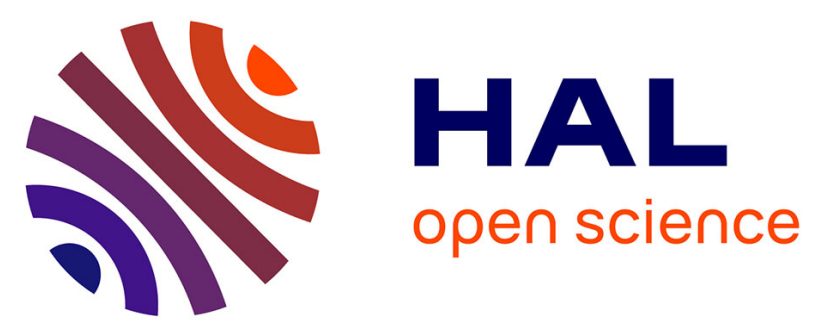

\title{
Identification par spectrométrie magnétique et mesure d'énergie
}

\author{
J.C. Jacmart, M. Liu, F. Mazloum, M. Riou, J.C. Roynette, C. Stephan
}

\section{To cite this version:}

J.C. Jacmart, M. Liu, F. Mazloum, M. Riou, J.C. Roynette, et al.. Identification par spectrométrie magnétique et mesure d'énergie. Revue de Physique Appliquée, 1969, 4 (2), pp.99-101. 10.1051/rphysap:019690040209900 . jpa-00243291

\section{HAL Id: jpa-00243291 https://hal.science/jpa-00243291}

Submitted on 1 Jan 1969

HAL is a multi-disciplinary open access archive for the deposit and dissemination of scientific research documents, whether they are published or not. The documents may come from teaching and research institutions in France or abroad, or from public or private research centers.
L'archive ouverte pluridisciplinaire HAL, est destinée au dépôt et à la diffusion de documents scientifiques de niveau recherche, publiés ou non, émanant des établissements d'enseignement et de recherche français ou étrangers, des laboratoires publics ou privés. 


\title{
IDENTIFIGATION PAR SPEGTROMÉTRIE MAGNÉTIQUE ET MESURE D'ÉNERGIE
}

\author{
J. G. JAGMART, M. LIU, F. MAZLOUM, \\ M. RIOU, J. G. ROYNETTE et G. STEPHAN, \\ Institut de Physique Nucléaire, 91-Orsay.
}

\begin{abstract}
Résumé. - Le principe et les caractéristiques de la méthode d'identification par spectrométrie magnétique et mesure d'énergie sont brièvement rappelés. Son utilisation avec trois jonctions à localisation et ordinateur I.B.M. 360 est décrite. Son application projetée à l'identification de noyaux "super-lourds " est présentée.

Abstract. - The principle and characteristics of the identification technique by magnetic spectrometry and energy measurement is briefly given. Its use with a three position solid state detectors and I.B.M. 360 computer is described. Its future application to the identification of "super-heavy" nuclei is presented.
\end{abstract}

L'application de cette méthode d'identification aux produits de réactions par ions lourds a déjà été décrite [1]. La mesure d'énergie sur une jonction après déflexion magnétique donne un spectre d'identification $E=\frac{(e B R)^{2}}{2} \times \frac{Z^{2}}{M}$. Rappelons brièvement que cette méthode présente deux avantages importants et un inconvénient : 1) une très bonne résolution sur le spectre d'identification $(0,4 \%)$ dans nos expériences (fig. 1) ; 2) un très faible fond parasite (de très bons résultats ont pu être obtenus dans l'axe du faisceau à $\left.0^{\circ}\right)$; 3) une ambiguïté sur l'état de charge des ions détectés qui, à l'énergie de l'ordre de $80 \mathrm{MeV}$ fournie par le cyclotron à énergie variable d'Orsay, n'est pas très gênante pour les noyaux plus légers que le fluor, mais rend cette méthode impropre à la détermination $\mathrm{du}$ numéro atomique des noyaux moyens et lourds.

Le spectromètre magnétique à double focalisation (indice $1 / 2$ ) a un rayon de $85 \mathrm{~cm}$ et un angle de déviation de $120^{\circ}$. Afin d'utiliser au mieux le plan focal, la mesure d'énergie est effectuée par trois jonctions à localisation de $5 \mathrm{~cm}$ de long, $1 \mathrm{~cm}$ de haut et $350 \mu$ d'épaisseur. Ceci permet de couvrir en une seule mesure une plage d'énergie $\Delta E / E=9 \%$. Après amplification et conversion digitale, les informations d'énergie et de position correspondant à chaque événement sont transmises directement à l'ordinateur I.B.M. 360 du laboratoire où elles sont, d'une part, enregistrées brutes sur bande magnétique et, d'autre part, rassemblées, après un premier traitement, sous forme de trois matrices de $1024 \times 10$ canaux (une pour chaque jonction) qui sont inscrites sur disque en fin de mesure. Une unité de visualisation (avec crayon lumineux) installée dans la salle de mesures permet de visualiser les projections de ces matrices [2]. On peut ainsi voir sur les trois jonctions la localisation correspondant à un noyau que l'on a identifié puis encadré au moyen $\mathrm{du}$ crayon lumineux sur les spectres d'identification; cette opération peut être effectuée soit sur la phase de mesure en cours d'acquisition, soit sur une des phases précédentes après avoir donné par machine à écrire son adresse sur le disque.

Afin de saisir l'intérêt de l'association de l'ordinateur à cette expérience, il faut se reporter à la figure 1 qui représente le spectre d'énergie (que nous appelons ici spectre d'identification) donné par une jonction à localisation pour une certaine valeur de l'induction dans le spectromètre. On constate pour tous les pics (correspondant chacun à un ion déterminé) une légère déformation provenant d'une part de la dispersion de l'aimant sur la longueur de la jonction, et d'autre part d'une réponse différente de celle-ci suivant la position. Après correction effectuée sur chaque événement par la calculatrice, on constate (en bas) une amélioration de la séparation. D'autre part, étant donné le nombre élevé de noyaux détectés simultanément (caractéristique des réactions entre ions lourds), seule une calculatrice en ligne permet d'utiliser pratiquement plusieurs jonctions à localisation en gardant un contrôle de l'expérience pendant l'acquisition des données.

Cet ensemble expérimental, techniquement assez lourd, ne peut être utilisé en permanence. En particulier, les réglages de l'électronique linéaire et les mesures à taux de comptage élevé (diffusion élastique) doivent être effectués sans liaison avec la calculatrice. On emploie, à cet effet, un analyseur Intertechnique 4096 canaux et un sélecteur de bande digital. Cet appareil, réalisé au laboratoire par M. J. Schiller à l'aide de micromodules, permet le réglage sans tâtonnement des seuils haut et bas à un canal près. Des 

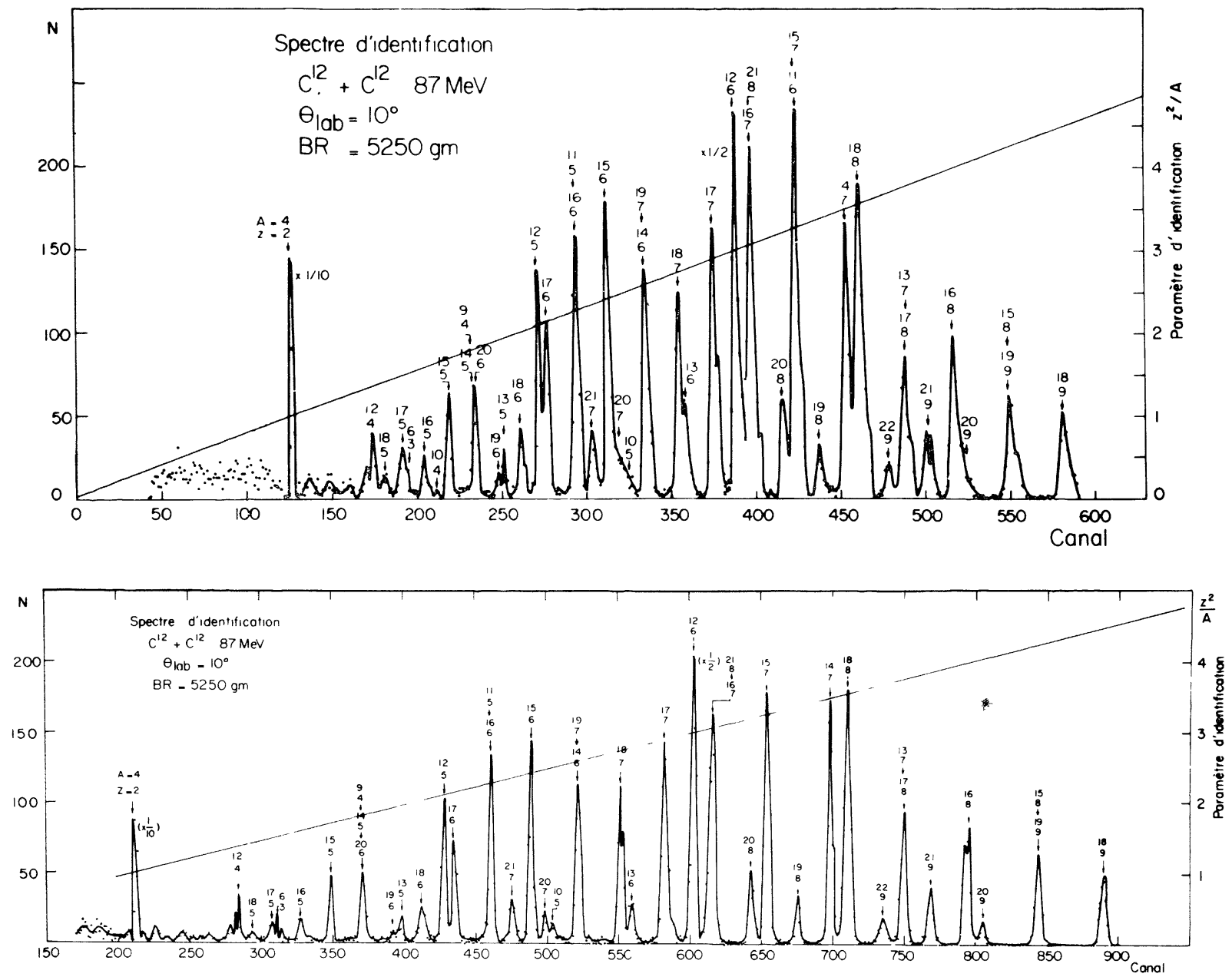

FIG. 1. - En haut : spectre d'énergie (ou d'identification) obtenu sur une jonction à localisation de $5 \mathrm{~cm}$ placée dans le plan focal du spectromètre (plage en énergie $\Delta E / E=3 \%$ ). En bas : le même spectre après correction, par la calculatrice, de la dispersion du spectromètre et de la réponse imparfaite de la jonction ( $A$ et $Z$ sont respectivement la masse et la charge de l'ion détecté).

spectres d'excitation et des distributions angulaires de réaction de transfert à un et plusieurs nucléons entre noyaux légers à une énergie de l'ordre de $80 \mathrm{MeV}$ (lab.) ont été obtenus avec cet ensemble expérimental [3].

Le principe de l'identification par spectrométrie et mesure d'énergie dont nous venons de voir les avantages sera utilisé à Orsay pour la recherche de nouveaux noyaux. En particulier, plusieurs auteurs [4] ont signalé l'existence possible d'un « îlot de stabilité » dans la région $Z=126$ ou 114 et $N=184$ ou 170 . Ces noyaux peuvent être formés par fusion de noyaux lourds à une énergie suffisamment élevée pour franchir la barrière coulombienne et fournir l'énergie de fusion nécessaire. Par exemple, les réactions :

$$
\begin{aligned}
& { }_{36}^{8: 2} \mathrm{Kr}+{ }_{90}^{230} \mathrm{Th} \rightarrow{ }_{126}^{312} \mathrm{X} \rightarrow{ }_{126}^{310} \mathrm{X}_{184}+2 \mathrm{n} \\
& { }_{36}^{86} \mathrm{Kr}+{ }_{78}^{198} \mathrm{Pt} \rightarrow{ }_{114}^{284} \mathrm{X}_{170}
\end{aligned}
$$

nécessitent une énergie incidente des ions $\mathrm{Kr}$ de l'ordre de $440 \mathrm{MeV}$ (lab.). L'ensemble Alice (injection au centre du cyclotron, où il seraient épluchés, d'ions accélérés à $1 \mathrm{MeV}$ par nucléon) permettra d'obtenir un tel faisceau [5]. Le noyau composé formé sera émis dans l'axe du faisceau avec une énergie de l'ordre de $115 \mathrm{MeV}$. Le spectromètre a l'avantage d'éliminer le faisceau. Son angle solide d'ouverture totale $3,5^{\circ}$ permet de détecter tous les événements, même après émission de quelques neutrons. L'état de charge le plus probable du noyau recherché sera de l'ordre de 25; en détectant successivement les différents états de charge à une même énergie, il sera possible de connaître ceux-ci. La masse pourra alors être déterminée avec la précision de la mesure sur $E(1 \%$ donnerait celle-ci à 3 unités près). Afin d'éliminer les risques de fausse identification provenant soit d'une diffusion du faisceau, soit de certains produits de 
fission, il sera nécessaire d'effectuer une mesure complémentaire de temps-de-vol : tous les parasites possibles auront un temps de transit dans le spectromètre plus court que celui du noyau recherché qui est $5 \times 10^{-7} \mathrm{~s}$. Ge temps donne la limite inférieure du temps de vie que celui-ci doit avoir pour être détecté.

Il faut remarquer que cette méthode aboutit à un « tri ». Après avoir détecté un produit intéressant, il est toujours possible, en gardant les mêmes conditions d'expérience, de modifier le détecteur pour étudier le noyau en question par l'une de ses caractéristiques (spectrométrie nucléaire et $\mathrm{X}$, chimie).

Nous remercions le service électronique, et en particulier MM. J. C. Brun, J. Schiller et G. Camatte, pour leur importante contribution, ainsi que M. J. P. Adam pour ses conseils dans l'élaboration du programme.

\section{BIBLIOGRAPHIE}

[1] JACMaR'T (J. C.) et al., Intern. Conf. on Heavy Ion Physics, Dubna (U.R.S.S.), 1966.

LIU (M.) et al., Session d'étude sur les méthodes d'identification, Lyon, janvier 1967.

[2] Adam (J. P.), Brun (J. C.) et Faucher (L.), ce colloque.

[3] von OeRTZEN (W.) et al., Phys. Letters, 1969, 28 B, 482.

LIU (M.) et al., Session d'études sur la physique et la production des ions lourds, La Plagne, mars 1969.
[4] MYers (W. D.) et Swatecki (W. J.), Nucl. Phys., 1966, 81, 1 et U.C.R.L. 11980.

Wong (C. Y.), Nucl. Phys., 1967, A 103, 625.

Rost (E.), Phys. Letters, 1968, 26 B, 184.

Pierce (T. E.) et Br.ANn (M.), Nucl. Phys., 1968, A 106, 14.

Nilsson (S. G.) et al., Nucl. Phys., 1968, A 115, 545.

[5] Cabrespine (A.), ce colloque. 